\title{
Tanshinone IIA suppresses ovarian cancer growth through inhibiting malignant properties and angiogenesis
}

\author{
Jin Zhou ${ }^{1 \#}$, Yuan-Yuan Jiang ${ }^{1 \#}$, Xiao-Xia Wang ${ }^{1}$, Hai-Ping Wang ${ }^{1}$, Huan Chen ${ }^{1}$, Yi-Chao Wu ${ }^{2}$, Long Wang ${ }^{1}$, \\ Xiang Pu ${ }^{1}$, Gui-Zhou Yue ${ }^{1}$, Li Zhang ${ }^{1}$ \\ ${ }^{1}$ College of Science, Sichuan Agricultural University, Ya'an, China; ${ }^{2}$ College of Life Science, China West Normal University, Nanchong, China \\ Contributions: (I) Conception and design: J Zhou, YY Jiang, L Zhang; (II) Administrative support: L Wang, X Pu, GZ Yue; (III) Provision of study \\ materials or patients: YY Jiang, L Zhang; (IV) Collection and assembly of data: XX Wang, HP Wang, H Chen; (V) Data analysis and interpretation: J \\ Zhou, YC Wu, L Zhang; (VI) Manuscript writing: All authors; (VII) Final approval of manuscript: All authors. \\ \#These authors contributed equally to this work. \\ Correspondence to: Li Zhang. College of Science, Sichuan Agricultural University, Ya'an 625014, China. Email: zhang8434@sina.com.
}

\begin{abstract}
Background: In Chinese herbal medicine, Tanshinone IIA (Tan-IIA) is one of the main compounds extracted from Salvia miltiorrbiza Bunge. Tan-IIA has been demonstrated to inhibit the growth of various tumors. However, the detailed molecular and cellular mechanisms of the antitumor effect of Tan-IIA have yet to be fully illuminated.

Methods: A2780 and ID- 8 were treated with $0,1.2,2.4,4.8$, or $9.6 \mu \mathrm{g} / \mathrm{mL}$ Tan-IIA for 24 hours. Cell counting Kit- 8 assay and EdU staining were used to evaluate cell proliferation. Terminal deoxynucleotidyl transferase dUTP nick end labeling (TUNEL) assay and flow cytometry were performed to analyze apoptosis. Western blot was carried out to determine the protein levels. Flow cytometry was used for cell cycle analysis. The levels of mRNA expression were analyzed by real-time polymerase chain reaction. The anti-tumor effect of Tan-IIA was observed in a tumor-bearing mouse model.

Results: Tan-IIA inhibited the proliferation of ovarian cancer cells in a dose-dependent manner by inducing G2/M phase arrest. It also down-regulated B-cell lymphoma 2 (Bcl-2) and up-regulated Bcl-2associated $\mathrm{X}$ protein (Bax) in ovarian cancer cells to induce apoptosis, and suppressed cell migration by inhibiting focal adhesion kinase phosphorylation. Tan-IIA significantly reduced vascular endothelial growth factor (VEGF) and cyclooxygenase-2 (COX2) mRNA expression in ovarian cancer cells. In vivo, Tan-IIA significantly inhibited tumor growth by inducing apoptosis and promoting anti-angiogenesis.
\end{abstract}

Conclusions: The results of this study shed light on the molecular and cellular mechanisms for the antitumor effect of Tan-IIA.

Keywords: Tanshinone IIA; ovarian cancer; tumor growth; angiogenesis

Submitted Jun 28, 2020. Accepted for publication Oct 16, 2020.

doi: $10.21037 / \mathrm{atm}-20-5741$

View this article at: http://dx.doi.org/10.21037/atm-20-5741

\section{Introduction}

Ovarian cancer is ranked as the seventh most common and the eighth deadliest malignancy among globally, with the 5 -year survival rate standing at less than $45 \%(1,2)$. In the majority of countries, ovarian cancer mortality rates have stabilized or fallen in the past two decades; however, in many low-income countries, they are rising. Current treatment regimens for ovarian cancer include surgery, chemotherapy and radiation therapy, and molecular targeted therapy $(3,4)$. Chemotherapy with alkylating agents, antimetabolites, antitumor antibiotics, platinum analogs, and natural anticancer agents is widely employed in the clinical treatment of ovarian cancer. The main reasons for the failure of chemotherapy are drug resistance and side effects. Therefore, the screening of high-efficiency and low toxicity antitumor drugs is urgently needed. Many 
anticancer drugs that originate from natural sources, such as paclitaxel, play important roles in chemotherapy (5-7).

Dan-shen, the dried root and rootstock of Salvia miltiorrhiza Bunge, is a crude drug that is a popular ingredient in many traditional Chinese medicines. Danshen is used to promote blood circulation, remove blood stasis, clear away toxic material, relieve restlessness, and eliminate edema in the treatment of numerous diseases $(8,9)$. Recent pharmacological studies found that the active ingredients of S. miltiorrhiza Bunge could expand the coronary artery, increase blood flow in the coronary artery, inhibit inflammation and inflection, and regulate immunity and antitumor activity (10-13). Tanshinone IIA (Tan-IIA) is among the principal active ingredients derived from Salvia miltiorrhiza radix. Tan-IIA is involved in antitumor activity in various cancers, such as colon cancer (14), malignant melanoma (15), lung cancer (16), and hepatocellular carcinoma (17). However, the full extent of the anticancer mechanisms of Tan-IIA has yet to be established.

In the present study, we analyzed the anti-tumor effects of Tan-IIA in vitro and in vivo and their molecular mechanisms. Our study found that Tan-IIA significantly induced cell proliferation by inducing G2/M phase arrest, promoted cell apoptosis through changing $\mathrm{B}$-cell lymphoma 2 (Bcl-2)/Bcl-2-associated X protein (Bax) balance, suppressed cell migration by reducing focal adhesion kinase (FAK) phosphorylation and blocking vascular endothelial growth factor (VEGF) expression. This study provided sufficient evidence that Tan-IIA could be an effective candidate agent in ovarian cancer therapy.

We present the following article in accordance with the ARRIVE reporting checklist (available at http://dx.doi. org/10.21037/atm-20-5741).

\section{Methods}

\section{Cell culture}

Human ovarian cancer cell line A2780 and mouse ovarian cancer cell line ID-8 were supplied by American Type Culture Collection (ATCC, USA). The cells were maintained in Dulbecco's Modified Eagle Medium (DMEM) containing $10 \%$ fetal bovine serum (FBS) and $1 \%$ penicillin/streptomycin (Gibco, USA) at $37^{\circ} \mathrm{C}$ with $5 \% \mathrm{CO}_{2}$ in a cell incubator.

\section{Cell counting Kit-8 assay (CCK-8)}

Cell viability was analyzed using the CCK- 8 assay kit (Beyotime, Shanghai, China) following the protocol of the manufacturer. A2780 and ID-8 cells were plated in 96-well culture plates and treated with Tan-IIA $(0,1.2,2.4,4.8$, or $9.6 \mu \mathrm{g} / \mathrm{mL}$ ). After 24 hours of treatment, $20 \mu \mathrm{L}$ of the CCK8 solution was added to the cells, before incubation at $37{ }^{\circ} \mathrm{C}$ for a further 2 hours. Each experiment was performed three times. The absorbency was measured at a test wavelength of $450 \mathrm{~nm}$ and a reference wavelength of $630 \mathrm{~nm}$.

\section{EdU staining assay}

EdU assay was performed using an EdU assay kit (Beyotime, Shanghai, China) following the protocol of the manufacturer. A2780 and ID-8 cells underwent treatment with Tan-IIA $(0,1.2,2.4,4.8$, or $9.6 \mu \mathrm{g} / \mathrm{mL})$ for 24 hours and then incubation with $50 \mathrm{mM} \mathrm{EdU}$ at $37{ }^{\circ} \mathrm{C}$. After incubation, the cells were fixed, permeabilized at room temperature, and washed two times with phosphatebuffered saline (PBS). EdU was added for 30 minutes, following which the cells were stained with Hoechest 33342 $(400 \mu \mathrm{L})$ for 30 minutes for visualization of the nuclei. The percentage of EdU-positive cells in different treatment groups were determined.

\section{Transwell assay}

For the cell migration assay, A2780 and mouse ID-8 cells underwent treatment with Tan- IA $(0,1.2,2.4,4.8$, or $9.6 \mu \mathrm{g} / \mathrm{mL})$. After treatment for 24 hours, the cells were placed in the upper chamber of each insert (Corning, Cambridge, USA), and $600 \mu \mathrm{L}$ DMEM medium (5\% FBS) was added to the lower chamber. The cells were incubated for 24 hours at $37^{\circ} \mathrm{C}$, then stained with $0.1 \%$ crystal violet. The numbers of migrated cells were counted under a microscope.

\section{Wound healing assay}

$2 \times 10^{5}$ A2780 were seeded into 6-well plates and cells influence reached $100 \%$, a scratch wound was created by scraping c, followed by three washes with serum-free medium to wash away nonadherent cells. The left cells were then underwent treatment with Tan-IIA $(0,1.2,2.4,4.8$, or $9.6 \mu \mathrm{g} / \mathrm{mL}$ ). Wounds were imaged at 0 and $24 \mathrm{~h}$ with microscope (Olympus, Shinjuku, Tokyo, Japan).

\section{Cell cycle analysis}

A2780 and mouse ID-8 cells underwent treatment with Tan-IIA $(0,1.2,2.4,4.8$, or $9.6 \mu \mathrm{g} / \mathrm{mL})$. The cells were 
Table 1 The primer sequences for real-time polymerase chain reaction

\begin{tabular}{lll}
\hline Gene & Primer sequences $(\mathrm{F})$ & Primer sequences (R) \\
\hline Human $\mathrm{BCl}-2$ & CGACGACTTCTCCCGCCGCTACCGC & CCGCATGCTGGGGCCGTACAGTTCC \\
Mouse $\mathrm{BCl}-2$ & CCTGTGCCACCATGTGTCCATC & GCTGAGAACAGGGTCTTCAGAGAC \\
Human Bax & TCCACCAAGAAGCTGAGCGAG & GTCCAGCCCATGATGGTTCT \\
Mouse Bax & GGTCCGGGGAGCAGCTTGGGAG & ACCACCTGCGTAGGACCCCGCC \\
Human VEGF & ATCTTCAAGCCATCCTGTGTGC & GCTCACCGCCTCGGCTTGT \\
Mouse VEGF & GAGCAGAAGTCCCATGAAGTG & CATGGTGATGTTGCTCTCTGA \\
Human COX2 & GCAAATCCTTGCTGTTCC & GGAGGAAGGGCTCTAGTA \\
Mouse COX2 & GGTGCCTGGTCTGATGATGTATG & ATGAGTATGAGTCTGCTGGTTTGG \\
Human GAPDH & TGCACCACCAACTGCTTAG & TAGAGGCAGGGATGATGTTC \\
Mouse GAPDH & GGTGAAGGTCGGTGTGACGGA & TGTTAATGGGGTCTCGCTCCTG \\
\hline
\end{tabular}

digested by EDTA free trypsin, before fixing with cold $70 \%$ ethanol for 12 hours at $4{ }^{\circ} \mathrm{C}$. The cells were collected at 2,000 $\mathrm{rpm} / \mathrm{min}$ for 5 minutes and resuspended in PBS (50 $\mu \mathrm{g} / \mathrm{mL}$ RNase A and $50 \mu \mathrm{g} / \mathrm{mL}$ propidium iodide), before incubation for 20 minutes at $37^{\circ} \mathrm{C}$. Cell cycle analysis was conducted using a flow cytometer.

\section{Terminal deoxynucleotidyl transferase dUTP nick end labeling assay}

Terminal deoxynucleotidyl transferase dUTP nick end labeling (TUNEL) assays were carried out, in line with the protocol of the manufacturer (Vazyme Biotech Co., Ltd., China). In the cell assay, A2780 and ID-8 cells underwent treatment with Tan-IIA $(0,1.2,2.4,4.8$, or $9.6 \mu \mathrm{g} / \mathrm{mL})$. After 24 hours of treatment, bright Red labeling mix was used to incubate the cells, which were then treated with Click-iT reaction cocktail. In the tissue assay, the sections of tumor tissue were incubated with Biotin-dUTP Labeling Mix, covered in Streptavidin-HRP for 30 minutes and then visualized with 3,3'-Diaminobenzidine (DAB) substrate. The number of TUNEL-positive cells and the apoptotic index were analyzed in each field.

\section{Western blotting assay}

Total protein from cells was extracted and quantified, and then separated with sodium dodecyl sulfate-polyacrylamide gel electrophoresis (SDS-PAGE) After separation, the protein was transferred to nitrocellulose (NC) membranes (Millipore, Bedford, MA, USA). Then, 5\% bovine serum albumin (BSA) in PBS containing Tween (PBST) was used to block the membranes for 1 hour, before incubation for 2 hours at $37^{\circ} \mathrm{C}$ with primary antibodies. The membranes were washed with PBST and incubated with secondary antibodies for $30 \mathrm{~min}$ at $37^{\circ} \mathrm{C}$. The protein were visualized by enhanced chemiluminescence (Millipore, Billerica, MA, USA). Protein expression was determined by ImageJ software and $\beta$-actin was used as a control. Ki67 $(1: 2,000)$, FAK $(1: 1,000)$, p-FAK $(1: 1,000)$, actin $(1: 4,000)$.

\section{Real-time reverse transcription-polymerase chain reaction}

TRIzol reagent (Invitrogen, Carlsbad, CA, USA) was used to extract total RNA from cells and tumors tissues in line with the instructions of the manufacturer. The First-Strand cDNA Synthesis Kit (Transgene, Beijing, China) was employed to reverse transcribe $1,000 \mathrm{ng}$ RNA into cDNA. Real-time polymerase chain reaction (PCR) was performed using 7500 Real-Time PCR System (Applied Biosystems, Foster City, CA, USA). Glyceraldehyde 3-phosphate dehydrogenase (GAPDH) was used as a control. The cycling program consisted of holding at $94^{\circ} \mathrm{C}$ for 2 minutes, then 30 cycles for $30 \mathrm{~s}$ at $94^{\circ} \mathrm{C}, 30 \mathrm{~s}$ at $56^{\circ} \mathrm{C}$, and $60 \mathrm{~s}$ at $72{ }^{\circ} \mathrm{C}$. The melting curve was collected to test PCR specificity. PCR analysis was carried out in triplicate. The relative mRNA expressions were calculated by the $2^{-\Delta \Delta c t}$ method. Table 1 displays the primer sequences used.

\section{Tumor xenograft model}

Five-week-old female BALB/c nude mice were supplied 
by Nanjing Model Animal Research Center. Experiments were performed under a project license (license number KS2020038) granted by the Animal Care Committee of Sichuan Agriculture University. Mouse research procedures in vivo were performed according to the guideline of the Animal Care Committee of Sichuan Agriculture University. A tumor xenograft model was established by injecting A2780 cells bilaterally and subcutaneously into the flanks of the mice $\left(100 \mu \mathrm{L}, 2 \times 10^{7}\right.$ cells). The mice were randomly divided into the control group and the Tan-IIA group $(30 \mathrm{mg} / \mathrm{kg})$, and administered an intraperitoneal injection of dimethyl sulfoxide (DMSO) or Tan-IIA, respectively.

\section{Immunobistochemistry assay}

The tissues were fixed and embedded in paraffin. The embedded sections were sliced into 6 - $\mu$ m-thick sections for immunohistochemistry assay. Immunohistochemical staining was performed with primary antibodies against Ki67, caspase-3, CD31, and VEGF. Next, the sections were incubated with horseradish peroxide-conjugated secondary antibody, visualized with DAB substrate, and counterstained with hematoxylin for detection. Images were photographed using the OLYMPUS microscope (OLYMPUS, Japan).

\section{Statistical analysis}

The data were presented as mean \pm SD and analyzed with SPSS 19.0 (Microsoft, USA). The Student's $t$-test or oneway analysis of variance (ANOVA) was used to analyze the data. A P value of $<0.05$ was considered to represent statistical significance.

\section{Results}

\section{Tan-IIA inbibited ovarian cancer cell proliferation by inducing G2/M phase arrest}

The effects of Tan-IIA $(0,1.2,2.4,4.8$, and $9.6 \mu \mathrm{g} / \mathrm{mL})$ on cell viability were assessed using EdU staining and CCK8 assay. The results of EdU staining indicated that after treatment with Tan-IIA, there was a significant dosedependent reduction in the percentage of EdU-positive cells $(\mathrm{P}<0.05)$ (Figure $1 A, B)$. The CCK-8 assay results showed that the viability of human A2780 and mouse ID-8 cells was significantly, dose-dependently inhibited $(\mathrm{P}<0.05)$ (Figure 1C). Western blot revealed that the Ki67 expression in A2780 and ID-8 cells was gradually, dose- dependently reduced after treatment with Tan-IIA $(\mathrm{P}<0.05)$ (Figure 1D,E). Previous studies have reported that Tan-I and Tan-IIA induce cell cycle arrest in multiple types of human cancer cells $(18,19)$. To investigate whether TanIIA induces cell cycle arrest in ovarian cancer cells, flow cytometry was carried out to analyze cell cycle distribution, showing that Tan-IIA induced G2/M cell phase arrest in a dose-dependent manner $(\mathrm{P}<0.05)$ (Figure 1F,G). These findings suggested that Tan-IIA could significantly inhibit the proliferation of ovarian cancer cells by inducing G2/M cell phase arrest.

\section{Tan-ILA induced apoptosis of ovarian cancer cells}

To confirm the apoptosis-inducing effects of Tan-IIA, TUNEL staining of A2780 and ID-8 cells was carried out. The cells were incubated for 24 hours in a growth medium with $0,1.2,2.4,4.8$, or $9.6 \mu \mathrm{g} / \mathrm{mL}$ Tan-IIA. The results of TUNEL staining demonstrated that there was a significant dose-dependent increase in TUNEL-positive A2780 and ID-8 cells (Figure $2 A, B$ ). Real-time PCR results showed that Bcl-2 mRNA expression was significantly down-regulated and Bax mRNA expression was significantly up-regulated in A2780 and ID-8 cells, in a dose-dependent manner $(\mathrm{P}<0.05)$ (Figure 2C,D). These results suggested that TanIIA promoted apoptosis by changing the balance of $\mathrm{Bcl}-2 /$ Bax.

\section{Tan-IIA blocked migration of ovarian cancer cells}

To better understand the effect of Tan-1 on cell migration, transwell assay and wound healing assay was carried out using cells. The results showed that Tan-IIA markedly and dose-dependently inhibited the migration of ovarian cancer cells $(\mathrm{P}<0.05)($ Figure $3 A, B)$. Wound healing assay revealed that Tan-IIA significantly inhibited wound healing in dose dependent manner $(\mathrm{P}<0.05)$ (Figure $3 C, D)$. The results of western blot showed that Tan-IIA did not affect the total FAK, but induced a significant, dose-dependent reduction in FAK phosphorylation $(\mathrm{P}<0.05)$ (Figure 3E,F).

\section{Tan-IIA inbibited the expression of VEGF and COX2 in ovarian cancer cells}

VEGF performs crucial activity in the growth of tumor blood vessel. Real-time PCR showed that Tan-IIA significantly, dose-dependently inhibited VEGF and cyclooxygenase-2 (COX2) mRNA expression by A2780 and 
A

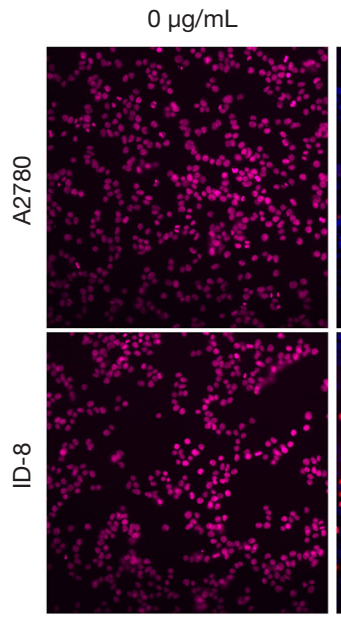

B

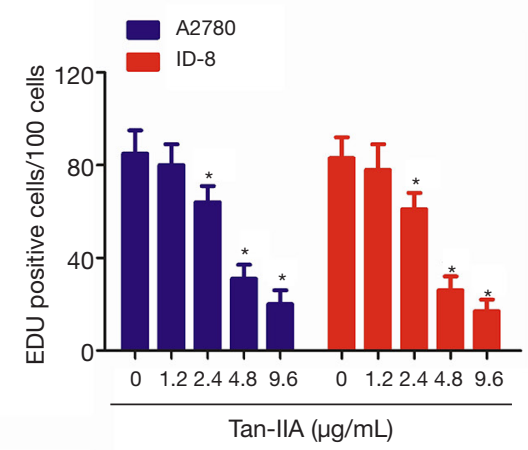

$E$

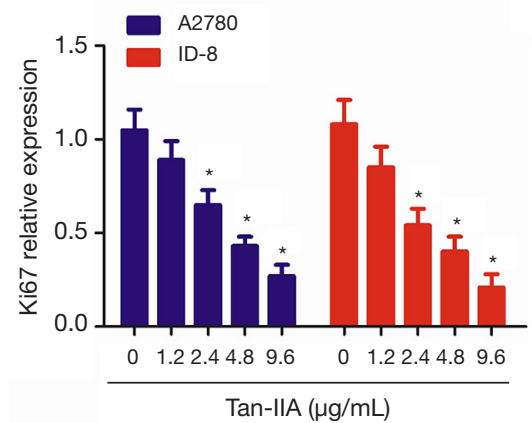

$1.2 \mu \mathrm{g} / \mathrm{mL}$

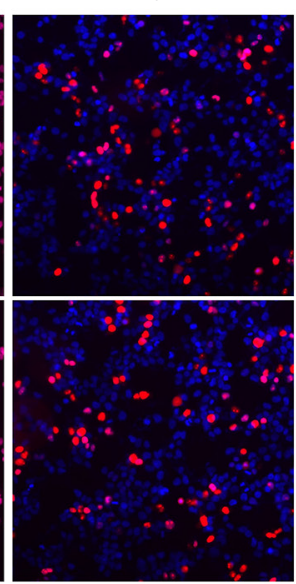

$2.4 \mu \mathrm{g} / \mathrm{mL}$

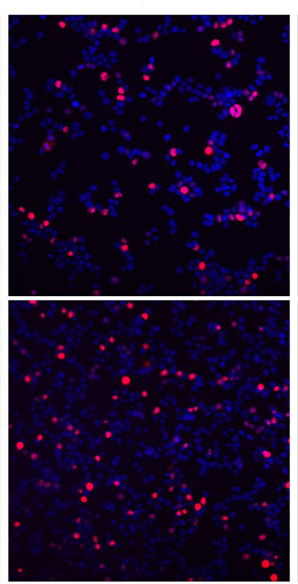

$4.8 \mu \mathrm{g} / \mathrm{mL}$

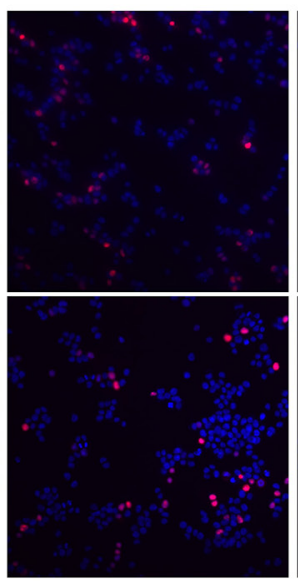

$9.6 \mu \mathrm{g} / \mathrm{mL}$

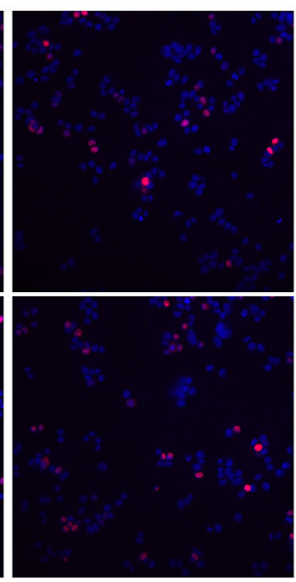

C

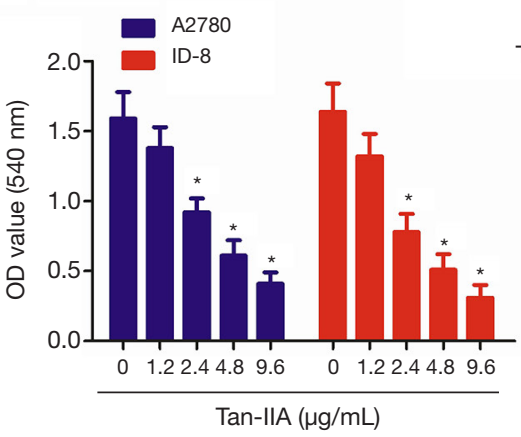

F

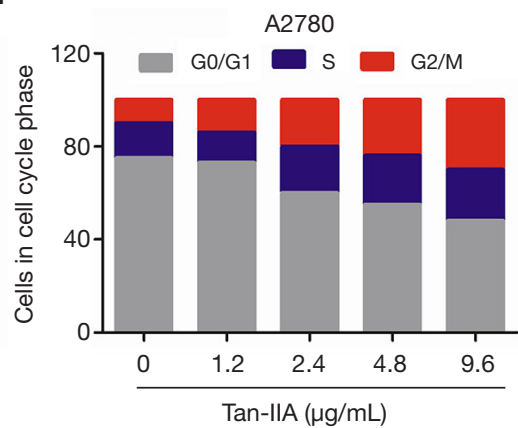

D

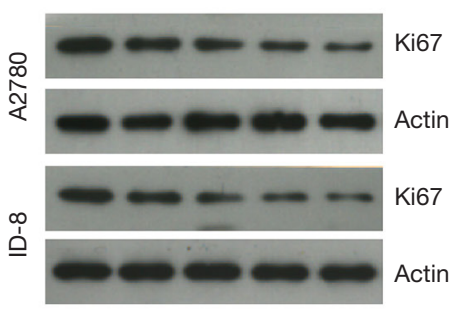

G

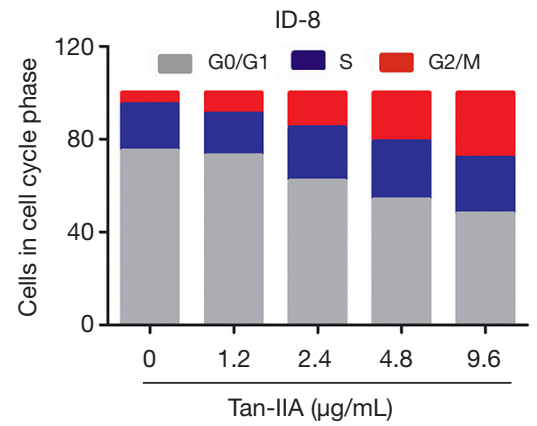

Figure 1 Tan-IIA inhibited the proliferation ability of ovarian cancer cells. (A) EdU staining. A2780 and ID-8 cells were treated with TanIIA at $1.2,2.4,4.8$, or $9.6 \mu \mathrm{g} / \mathrm{mL}$ for 24 hours, and stained with EdU, amplification $100 \times$. (B) The percentages of EdU-positive A2780 and ID-8 cells. (C) Cell counting Kit-8 (CCK-) assay. A2780 and ID-8 cells were treated with Tan-IIA at 1.2, 2.4, 4.8, or 9.6 $\mu \mathrm{g} / \mathrm{mL}$ for 24 hours and stained with CCK-8 kit. (D,E) Ki67 protein expression. A2780 and ID-8 cells were treated with Tan-IIA at 1.2, 2.4, 4.8, or 9.6 $\mu$ g/mL for 24 hours and analyzed by western blot. (F,G) Cell cycle distribution. A2780 and ID-8 cells were treated with Tan-IIA at 1.2, 2.4, 4.8, or $9.6 \mu \mathrm{g} / \mathrm{mL}$ for 24 hours. Cell cycle distribution was analyzed by flow cytometry. * $\mathrm{P}<0.05$. 

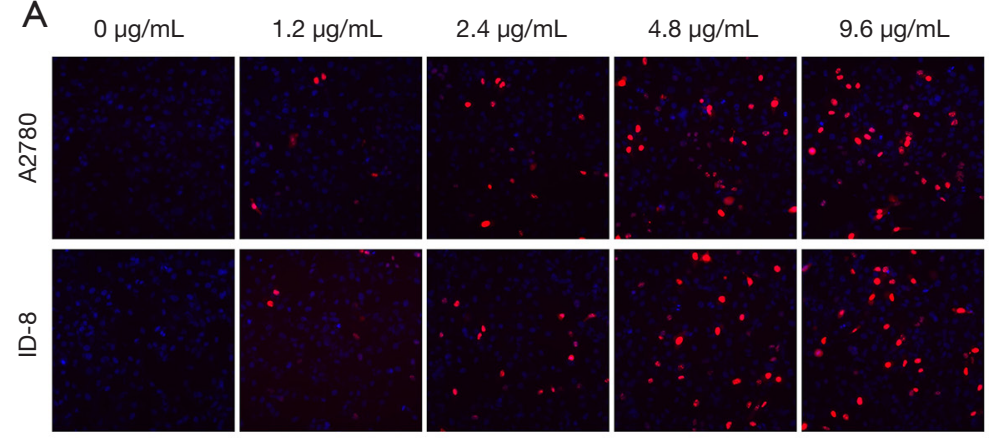

C

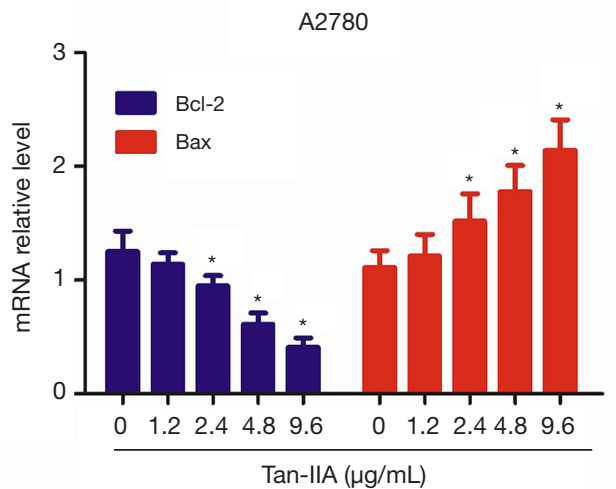

B

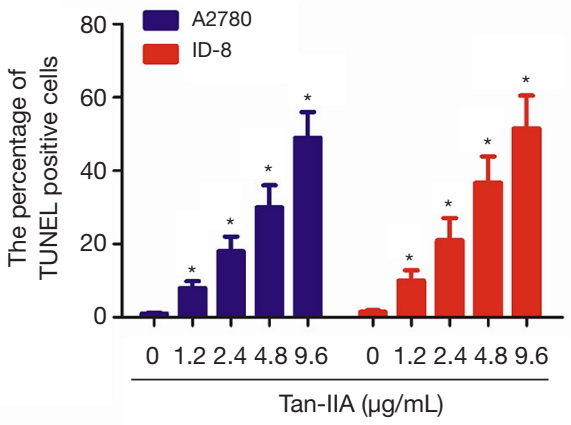

ID-8
D

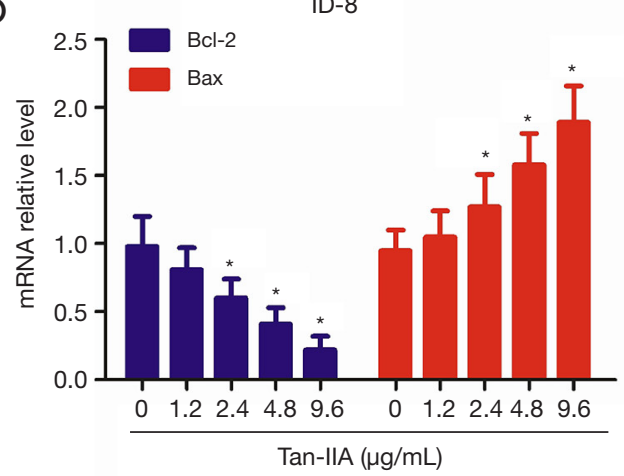

Figure 2 Tan-IIA induced apoptosis in ovarian cancer cells. (A) Apoptosis assay. A2780 and ID-8 cells were treated with Tan-IIA at 1.2, 2.4, 4.8, or $9.6 \mu \mathrm{g} / \mathrm{mL}$ for 24 hours and analyzed by terminal deoxynucleotidyl transferase dUTP nick end labeling staining. Amplification 100x. (B) The rates of apoptosis in A2780 and ID-8 cells. (C,D) Bcl-2 and Bax mRNA expression level in A2780 and ID-8 cells by real-time polymerase chain reaction. * $\mathrm{P}<0.05$.

ID-8 cells $(\mathrm{P}<0.05)$ (Figure 4A,B).

\section{Tan-ILA inbibited tumor growth in vivo}

To explore the antitumor effect of Tan-IIA in vivo, a tumor xenograft mouse model was established by injecting ID-8 cells into the flanks of nude mice. The tumor weight in the Tan-IIA group significantly reduced compared with the control group $(\mathrm{P}<0.05)$ (Figure $5 A, B)$. The results of immunohistochemistry assay indicated that the Ki67 expression in the Tan-II group was significantly reduced compared to that in the control group $(\mathrm{P}<0.05)$ (Figure $5 C)$, The tumor apoptotic index in Tan-IIA group was significantly higher than that in the control group $(\mathrm{P}<0.05)$ (Figure 5D). Additionally, the protein expression levels of VEGF and COX2 in the Tan-IIA group were significantly down-regulated in comparison with the control group $(\mathrm{P}<0.05)$ (Figure 5E,F). Also, the tumor vascular density in the Tan-IIA group was significantly decreased compared with that in the control group $(\mathrm{P}<0.05)$ (Figure $5 G)$. These results showed that Tan-IIA inhibited tumor growth through inducing apoptosis and suppressing tumor angiogenesis.

\section{Discussion}

Tanshinone IIA (Tan-IIA) has shown anti-cancer activity in various cancers. In this study, we explored the biological function and mechanisms of Tan-IIA in ovarian cancer. We demonstrated that Tan-IIA effectively inhibited the proliferation and migration abilities of A2780 and ID-8 cells, and induced A2780 and ID-8 cell apoptosis. Additionally, Tan-IIA significantly inhibited tumor growth by tumor angiogenesis in vivo by down-regulating VEGF and COX2 expression.

Previous studies have shown that Tan-IIA can inhibit the proliferation of tumor cells, including malignant astrocytoma (20), non-small cell lung cancer (NSCLC) (21), 
A

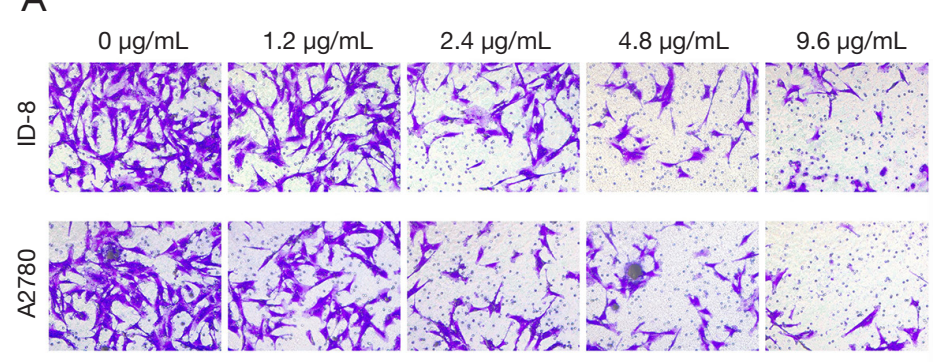

C

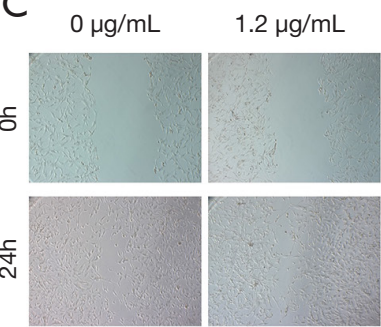

$\mathrm{E}$

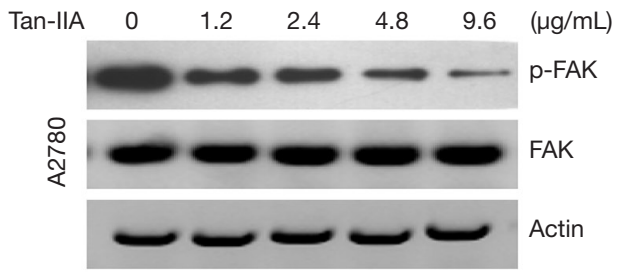

B

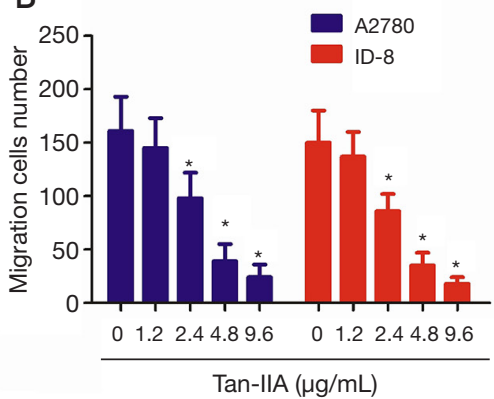

$\mathrm{D}$

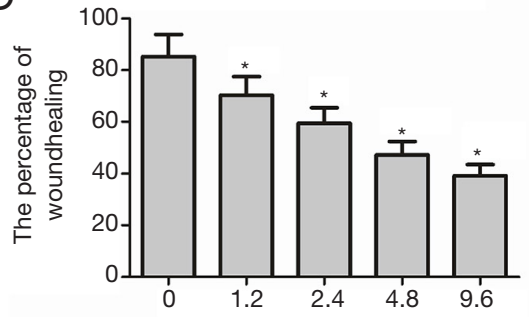

$\mathrm{F}$

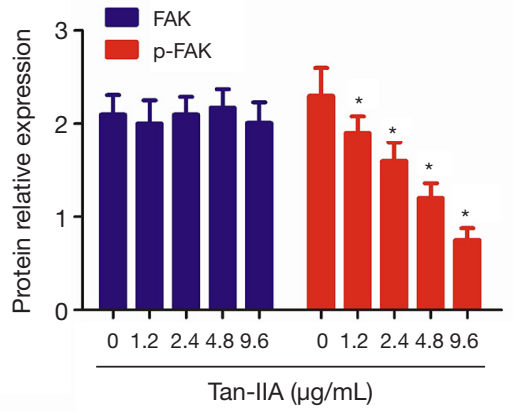

Figure 3 Tan-IIA inhibited the migration ability of ovarian cancer cells. (A) Cell migration assay. A2780 and ID-8 cells were treated with Tan-IIA at 1.2, 2.4, 4.8, or $9.6 \mu \mathrm{g} / \mathrm{mL}$ for 24 hours, and analyzed by Transwell assay. Amplification 100x. (B) The number of migrated A2780 and ID-8 cells in (A). (C and D) Wound healing assay. A2780 were treated with Tan-IIA at 1.2, 2.4, 4.8, or $9.6 \mu \mathrm{g} / \mathrm{mL}$ for 24 hours, and analyzed by wound healing assay. (E and F) Focal adhesion kinase protein expression in A2780 and ID-8 cells by Western blot. *, P<0.05.
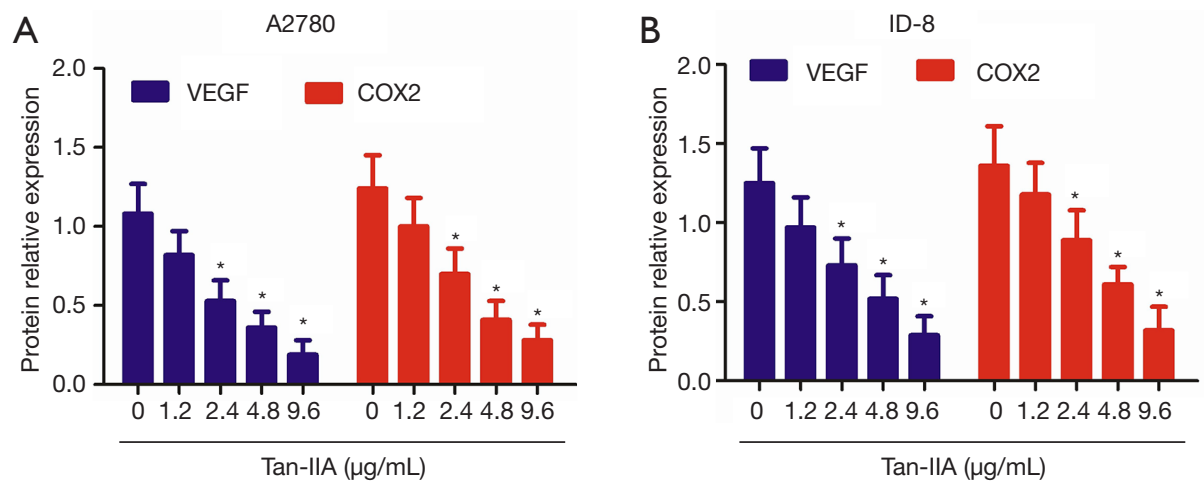

Figure 4 Tan-IIA inhibited vascular endothelial growth factor and cyclooxygenase-2 expression in ovarian cancer cells. (A) The mRNA expressions of vascular endothelial growth factor (VEGF) and cyclooxygenase-2 (COX2) in A2780 after Tan-IIA treatment at 1.2, 2.4, 4.8, or $9.6 \mu \mathrm{g} / \mathrm{mL}$ for 24 hours by real-time polymerase chain reaction (PCR). (B) VEGF and COX2 mRNA expression in ID-8 after Tan-IIA treatment at $1.2,2.4,4.8$, or $9.6 \mu \mathrm{g} / \mathrm{mL}$ for 24 hours by real-time $\mathrm{PCR} .{ }^{*}, \mathrm{P}<0.05$. 
A

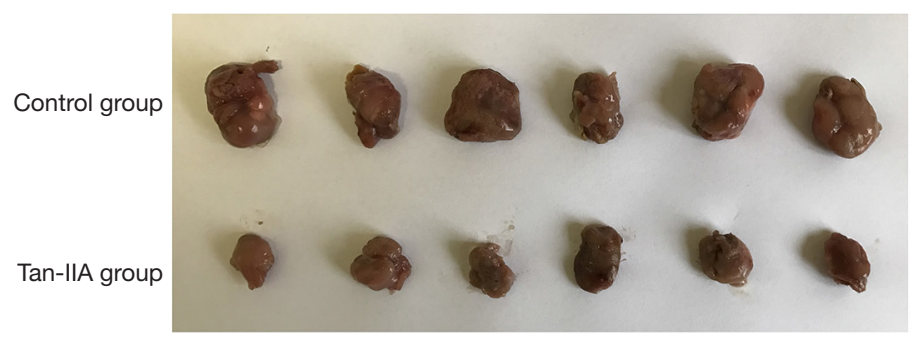

B

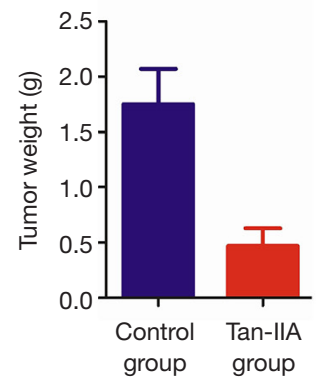

C

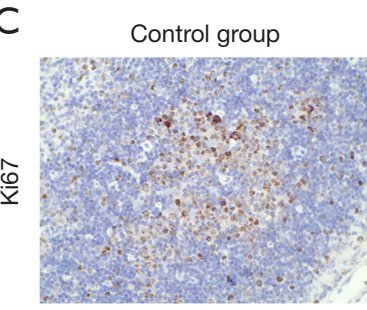

E

峛

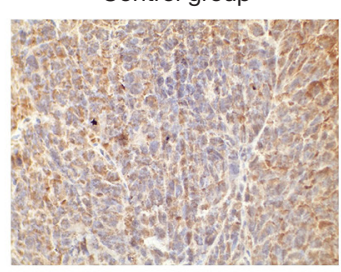

Tan-IIA group

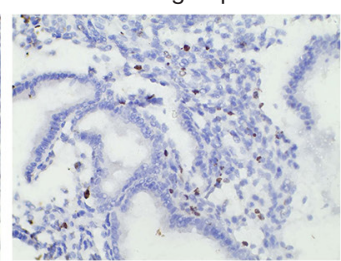

Tan-IIA group

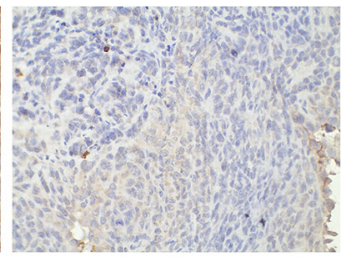

D

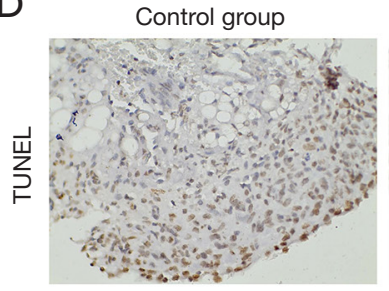

$\mathrm{F}$

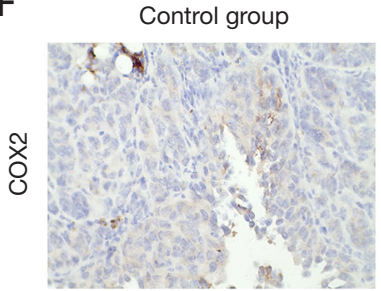

Tan-IIA group

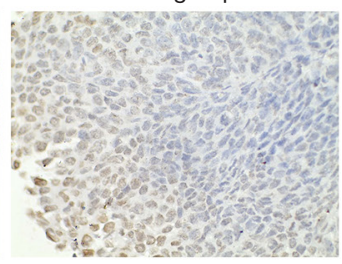

Tan-IIA group

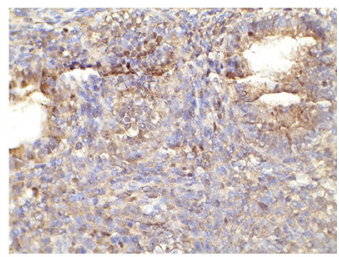

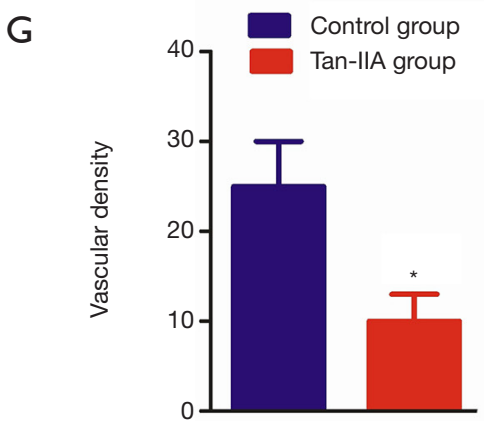

Figure 5 Tan-IIA inhibited tumor growth in vivo. (A) Tumors in the control group and the Tan-IIA group were isolated and pictured after 4 weeks of treatment; (B) the tumors of the control group and Tan-IIA group mice were weighed; (C) Ki67 protein expression was detected by immunohistochemistry in the two groups of mice. Amplification 100x; (D) tumor cell apoptosis in the control and Tan-IIA groups was analyzed by terminal deoxynucleotidyl transferase dUTP nick end labeling staining. Amplification 100x; (E) vascular endothelial growth factor protein expression was analyzed by immunohistochemistry in the two groups of mice. Amplification 100x; (F) cyclooxygenase-2 protein expression was analyzed by immunohistochemistry in the two groups of mice. Amplification 100x; (G) the number of tumor vessels in the two groups was analyzed by CD31 immunohistochemistry. *, $\mathrm{P}<0.05$. 
colorectal cancer (22), and gastric cancer (23). Our study firstly demonstrated that Tan-IIA suppressed proliferation ability of ovarian cancer cells. An earlier report showed that Tan-I significantly inhibited cell progression through the $\mathrm{S}$ and $\mathrm{G} 2$ phases of the cell cycle by down-regulating the expressions of cyclin A and cyclin B (24). Other studies demonstrated that Tan-IIA treatment caused $\mathrm{S}$ phase arrest in 786-O cells (19) and A549 cells (25). Ma et al. found that Tan-IIA induces G0/G1-phase cell-cycle arrest in NSCLC cell line H1299 (26). Our study on ovarian cancer cells demonstrated that G2/M phase arrest was induced by TanIIA. Controversy still surrounds the effects of Tan-IIA on the cell cycle. Therefore, we propose that different doses of Tan-IIA affect the cell cycles of various cells differently.

Additional, earlier reports showed that Tan-IIA induced cell apoptosis in different cancer cells by different molecular mechanisms. He et al. showed that Tan-IIA regulates the apoptosis of colorectal cancer cells through attenuating Parkin-mediated mitophagy via the suppression of the AMPK/Skp2 pathways (27). Zhang et al. showed that Tan IIA can induce apoptosis in human esophageal carcinoma Eca-109 cells through regulating cytochrome c and caspase-9 expression (28). The results of this study showed that Tan-IIA significantly induced cell apoptosis by changing the Bcl-2/Bax ratio in ovarian cancer cells.

Previous studies have also shown that Tan-IIA suppresses the migration of cervix carcinoma stem cells by inhibiting the transcriptional activity of yes-associated protein (29), inhibits gastric cancer cell migration by downregulating FOXM1 (23), and inhibits human colon cancer cell migration by downregulating the expressions of mTOR protein and VEGF mRNA (14). We disclosed a new mechanism of Tan-IIA in ovarian cancer, in which it has an inhibitive effect on cell migration. Western blot showed that Tan-IIA reduced FAK phosphorylation in a dose-dependent manner. FAK is a non-receptor tyrosine kinase that plays a role in regulating cell migration and invasion in many cancer cells $(30,31)$. Some studies have reported that the downregulation of FAK or inhibition of FAK kinase activity can reduce the migration and invasion abilities of human conventional renal cell carcinoma and esophageal squamous cell carcinoma cells $(32,33)$. Tan-IIA down-regulated FAK phosphorylation to inhibit cancer cell migration.

Tumor angiogenesis also performs pivotal activity in promoting the differentiation and proliferation of tumor cells, and is an important pathway for the infiltration, invasion, and distant metastasis of tumor cells (34-36). The formation of tumor angiogenesis is regulated by many angiogenic growth factors, such as VEGF, basic fibroblast growth factor (bFGF), and COX2 (37-39). This study showed that Tan-IIA significantly, dose-dependently reduced VEGF and COX2 protein expression in ovarian cancer cells. In our tumor mouse model, Tan-IIA inhibited tumor vessel density by down-regulating VEGF protein expression.

In summary, this study provides robust evidence to support the antitumor effect of Tan-IIA in ovarian cancer. In vitro and in vivo experiments showed that Tan-IIA arrested the G2/M phase cell cycle, induced cell apoptosis, and inhibited cell migration and tumor angiogenesis. The results showed Tan-IIA to be a potential anticancer agent for ovarian cancer therapy.

\section{Acknowledgments}

Funding: This study was supported by Sichuan Science and Technology Program (20ZDYF1286, 2019YFS0021), Sichuan Crops and Animals Breeding Special Project (2016NYZ0036), Sichuan Agricultural University (03572198, 03572098, 03571541), and the National Modern Agricultural Industry Technology System Sichuan Innovation Team (SCCXTD-2020-19).

\section{Footnote}

Reporting Checklist: The authors have completed the ARRIVE reporting checklist. Available at http://dx.doi. org/10.21037/atm-20-5741

Data Sharing Statement: Available at http://dx.doi. org/10.21037/atm-20-5741

Conflicts of Interest: All authors have completed the ICMJE uniform disclosure form (available at http://dx.doi. org/10.21037/atm-20-5741). The authors have no conflicts of interest to declare.

Ethical Statement: The authors are accountable for all aspects of the work in ensuring that questions related to the accuracy or integrity of any part of the work are appropriately investigated and resolved. Experiments were performed under a project license (license number KS2020038) granted by the Animal Care Committee of Sichuan Agriculture University. Mouse research procedures in vivo were performed according to the guideline of the Animal Care Committee of Sichuan Agriculture University. 
Open Access Statement: This is an Open Access article distributed in accordance with the Creative Commons Attribution-NonCommercial-NoDerivs 4.0 International License (CC BY-NC-ND 4.0), which permits the noncommercial replication and distribution of the article with the strict proviso that no changes or edits are made and the original work is properly cited (including links to both the formal publication through the relevant DOI and the license). See: https://creativecommons.org/licenses/by-nc-nd/4.0/.

\section{References}

1. Webb PM, Jordan SJ. Epidemiology of epithelial ovarian cancer. Best Pract Res Clin Obstet Gynaecol 2017;41:3-14.

2. Marchetti C, Rosati A, Scambia G, et al. Secondary cytoreduction in platinum-sensitive recurrent ovarian cancer: are we missing something? Ann Transl Med. 2019;7:S372.

3. Cortez AJ, Tudrej P, Kujawa KA, et al. Advances in ovarian cancer therapy. Cancer Chemother Pharmacol 2018;81:17-38.

4. Xue C, Zhu D, Chen L, et al. Expression and prognostic value of $\mathrm{PD}-\mathrm{L} 1$ and $\mathrm{PD}-\mathrm{L} 2$ in ovarian cancer. Transl Cancer Res 2019;8:111-9.

5. Chan JK, Brady MF, Penson RT, et al. Weekly vs. Every3-week paclitaxel and carboplatin for ovarian cancer. N Engl J Med 2016;374:738-48.

6. Richardson DL, Coleman RL, Sill MW. Paclitaxel and pazopanib in ovarian cancer-reply. JAMA Oncol 2018;4:1299.

7. Bojko A, Czarnecka-Herok J, Charzynska A, et al. Diversity of the senescence phenotype of cancer cells treated with chemotherapeutic agents. Cells 2019;8:1501.

8. Su CY, Ming QL, Rahman K, et al. Salvia miltiorrhiza: Traditional medicinal uses, chemistry, and pharmacology. Chin J Nat Med 2015;13:163-82.

9. MEIm XD, Cao YF, Che YY, et al. Danshen: a phytochemical and pharmacological overview. Chin J Nat Med 2019;17:59-80.

10. Wang L, Ma R, Liu C, et al. Salvia miltiorrhiza: A potential red light to the development of cardiovascular diseases. Curr Pharm Des 2017;23:1077-97.

11. Chung YC, Hsieh FC, Lin YJ, et al. Magnesium lithospermate $\mathrm{b}$ and rosmarinic acid, two compounds present in salvia miltiorrhiza, have potent antiviral activity against enterovirus 71 infections. Eur J Pharmacol 2015;755:127-33.

12. Gao H, Huang L, Ding F, et al. Simultaneous purification of dihydrotanshinone, tanshinone i, cryptotanshinone, and tanshinone IIA from salvia miltiorrhiza and their antiinflammatory activities investigation. Sci Rep 2018;8:8460.

13. Chen X, Guo J, Bao J, et al. The anticancer properties of salvia miltiorrhiza bunge (danshen): A systematic review. Med Res Rev 2014;34:768-94.

14. Xue J, Jin X, Wan X, et al. Effects and mechanism of tanshinone ii a in proliferation, apoptosis, and migration of human colon cancer cells. Med Sci Monit 2019;25:4793-800.

15. Li X, Li Z, Li X, et al. Mechanisms of tanshinone ii a inhibits malignant melanoma development through blocking autophagy signal transduction in a375 cell. BMC Cancer 2017;17:357.

16. Au Yeung CL, Co NN, Tsuruga T, et al. Exosomal transfer of stroma-derived mir21 confers paclitaxel resistance in ovarian cancer cells through targeting apaf1. Nat Commun 2016;7:11150.

17. Hu Y, Wang $\mathrm{S}, \mathrm{Wu} \mathrm{X}$, et al. Chinese herbal medicinederived compounds for cancer therapy: A focus on hepatocellular carcinoma. J Ethnopharmacol 2013;149:601-12.

18. Tian QT, Ding CY, Song SS, et al. New tanshinone i derivatives s222 and s439 similarly inhibit topoisomerase $\mathrm{i} / \mathrm{ii}$ but reveal different $\mathrm{p} 53$-dependency in inducing $\mathrm{g} 2 / \mathrm{m}$ arrest and apoptosis. Biochem Pharmacol 2018;154:255-64.

19. Wei X, Zhou L, Hu L, et al. Tanshinone IIA arrests cell cycle and induces apoptosis in 786-o human renal cell carcinoma cells. Oncol Lett 2012;3:1144-8.

20. Dong W, Zhang Y, Chen X, et al. High-dose tanshinone IIA suppresses migration and proliferation while promoting apoptosis of astrocytoma cells via notch-1 pathway. Neurochem Res 2018;43:1855-61.

21. Xie J, Liu JH, Liu H, et al. Tanshinone IIA combined with adriamycin inhibited malignant biological behaviors of nsclc a549 cell line in a synergistic way. BMC Cancer 2016;16:899.

22. Jieensinue S, Zhu H, Li G, et al. Tanshinone IIA reduces sw837 colorectal cancer cell viability via the promotion of mitochondrial fission by activating jnk-mff signaling pathways. BMC Cell Biol 2018;19:21.

23. Yu J, Wang X, Li Y, et al. Tanshinone IIA suppresses gastric cancer cell proliferation and migration by downregulation of foxm1. Oncol Rep 2017;37:1394-400.

24. Tung YT, Chen HL, Lee CY, et al. Active component of danshen (salvia miltiorrhiza bunge), tanshinone i, attenuates lung tumorigenesis via inhibitions of vegf, cyclin a, and cyclin b expressions. Evid Based Complement 
Alternat Med 2013;2013:319247.

25. Xie J, Liu J, Liu H, et al. The antitumor effect of tanshinone IIA on anti-proliferation and decreasing vegf/ vegfr2 expression on the human non-small cell lung cancer a549 cell line. Acta Pharm Sin B 2015;5:554-63.

26. Ma ZL, Zhang BJ, Wang DT, et al. Tanshinones suppress aurka through up-regulation of mir-32 expression in nonsmall cell lung cancer. Oncotarget 2015;6:20111-20.

27. He L, Gu K. Tanshinone IIA regulates colorectal cancer apoptosis via attenuation of parkinmediated mitophagy by suppressing ampk/skp2 pathways. Mol Med Rep 2018;18:1692-703.

28. Zhang Y, Li S, He H, et al. Influence of tanshinone IIA on apoptosis of human esophageal carcinoma eca109 cells and its molecular mechanism. Thorac Cancer 2017;8:296-303.

29. Qin J, Shi H, Xu Y, et al. Tanshinone IIA inhibits cervix carcinoma stem cells migration and invasion via inhibiting yap transcriptional activity. Biomed Pharmacother 2018;105:758-65.

30. Lee BY, Timpson P, Horvath LG, et al. Fak signaling in human cancer as a target for therapeutics. Pharmacol Ther 2015;146:132-49.

31. Zhao X, Guan JL. Focal adhesion kinase and its signaling pathways in cell migration and angiogenesis. Adv Drug Deliv Rev 2011;63:610-5.

32. Béraud C, Dormoy V, Danilin S, et al. Targeting fak

Cite this article as: Zhou J, Jiang YY, Wang XX, Wang HP, Chen H, Wu YC, Wang L, Pu X, Yue GZ, Zhang L. Tanshinone IIA suppresses ovarian cancer growth through inhibiting malignant properties and angiogenesis. Ann Transl Med 2020;8(20):1295. doi: 10.21037/atm-20-5741 scaffold functions inhibits human renal cell carcinoma growth. Int J Cancer 2015;137:1549-59.

33. Zhu X, Wang J, Li L, et al. Gpx3 suppresses tumor migration and invasion via the fak/akt pathway in esophageal squamous cell carcinoma. Am J Transl Res 2018;10:1908-20.

34. Ronca R, Benkheil M, Mitola S, et al. Tumor angiogenesis revisited: Regulators and clinical implications. Med Res Rev 2017;37:1231-74.

35. De Palma M, Biziato D, Petrova TV. Microenvironmental regulation of tumour angiogenesis. Nat Rev Cancer 2017;17:457-74.

36. Hida K, Maishi N, Annan DA, et al. Contribution of Tumor Endothelial Cells in Cancer Progression. Int J Mol Sci 2018;19:1272.

37. Kujawski M, Kortylewski M, Lee H, et al. Stat3 mediates myeloid cell-dependent tumor angiogenesis in mice. J Clin Invest 2008;118:3367-77.

38. Salvado MD, Alfranca A, Haeggstrom JZ, et al. Prostanoids in tumor angiogenesis: Therapeutic intervention beyond cox-2. Trends Mol Med 2012;18:233-43.

39. Eswarappa SM, Fox PL. Antiangiogenic vegf-ax: A new participant in tumor angiogenesis. Cancer Res 2015;75:2765-9.

(English Language Editor: J. Reynolds) 